\title{
Plasmodium falciparum Acetyl-CoA Synthetase is essential for parasite intraerythrocytic development and chromatin modification
}

Isadora Oliveira Prata ${ }^{\mathrm{a}}$, Eliana Fernanda Galindo Cubillos ${ }^{\mathrm{a}}$, Arne Krüger ${ }^{\mathrm{a}}$, Deibs Barbosa $^{\mathrm{b}}$, Joaquim Martins Juniorb, João Carlos Setubal ${ }^{\mathrm{b}}$, Gerhard Wunderlich, ${ }^{\mathrm{a}}{ }^{*}$

a Department of Parasitology, Institute for Biomedical Sciences, University of São Paulo, Avenida Professor Lineu Prestes, 1374, 05508-000, São Paulo-SP, Brazil.

b Department of Biochemistry, Institute of Chemistry, University of São Paulo, Avenida Professor Lineu Prestes, 748, 05508-000, São Paulo-SP, Brazil.

* To whom correspondence should be sent, gwunder@usp.br

This file contains Supporting information for the manuscript.

Supporting Figure S1: Map of the transfection plasmid.

Supporting Figure S2: Fluorescence microscopy of ring stage parasites showing ACSGFP expression.

Supporting Figure S3: Fluorescence microscopy of trophozoite stage parasites showing ACS-GFP expression.

Supporting Figure S4: Western blot showing ACS-GFP intensities in control and knocked down or drug-treated parasites.

Supporting Figure S5: Cytotoxicity test of iACS against HepG2 cells.

Supporting Table S1: Table in Excel format showing the complete output of the ChIPseq analysis.

Supporting Table S2: Table in Excel format showing the filtered output of the ChIPseq analysis.

Supporting Table S3:Table in Excel format showing the primers used for var gene transcript analysis. These primers were previously published by Salanti and colleagues (cited in the main text).

Supporting Table S4: This table in Excel format shows the results of the GO term enrichment analysis with loci to which ACS significantly associated (done in PlasmoDB). 


\section{Figure S1}

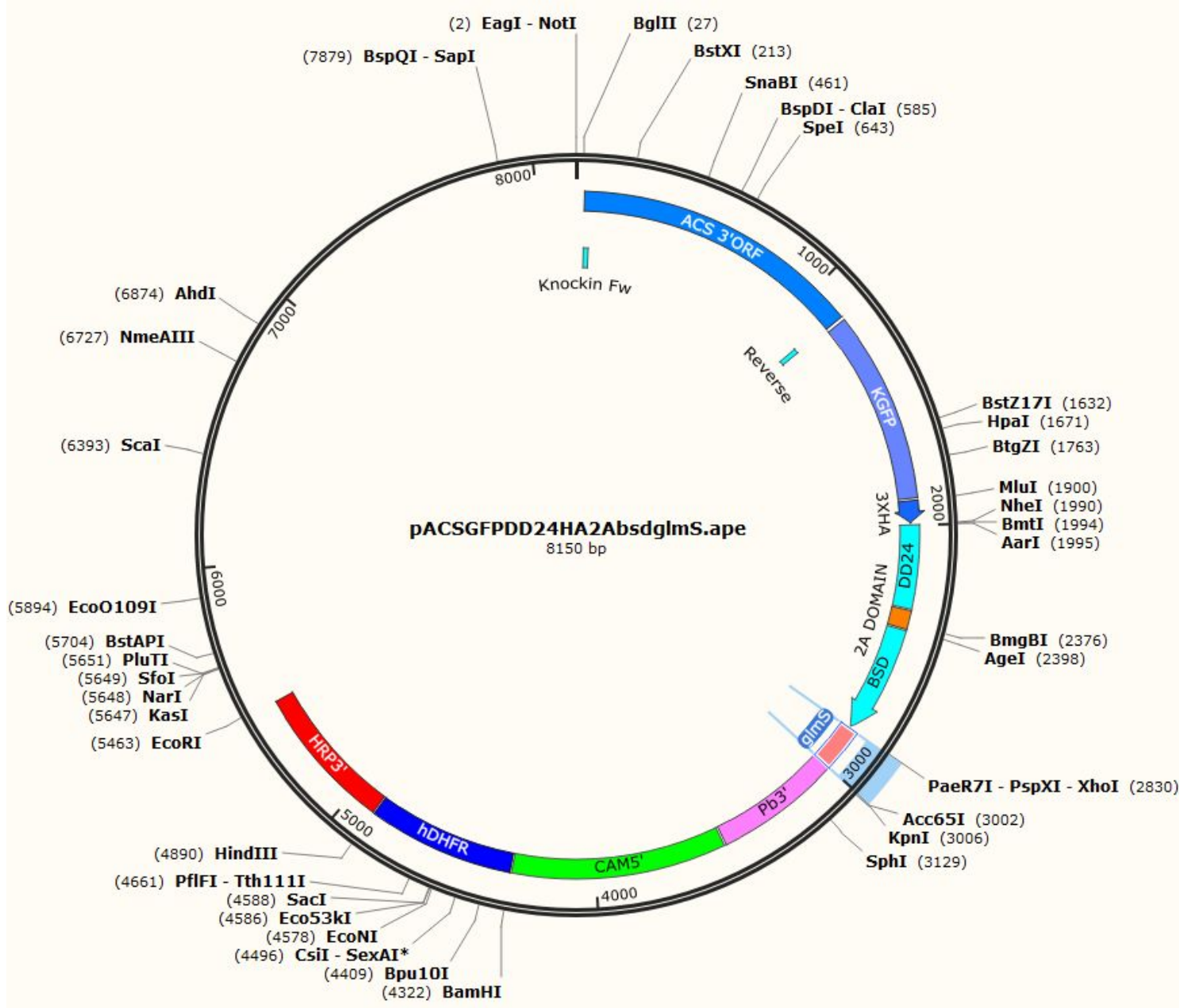

Figure S1 - Map of the pACS-GFP-HA-DD24-2A-BSD-glmS vector construct. The vector sequence is available upon request (gwunder@usp.br). The map was created using APE software (https://jorgensen.biology.utah.edu/wayned/ape/). 

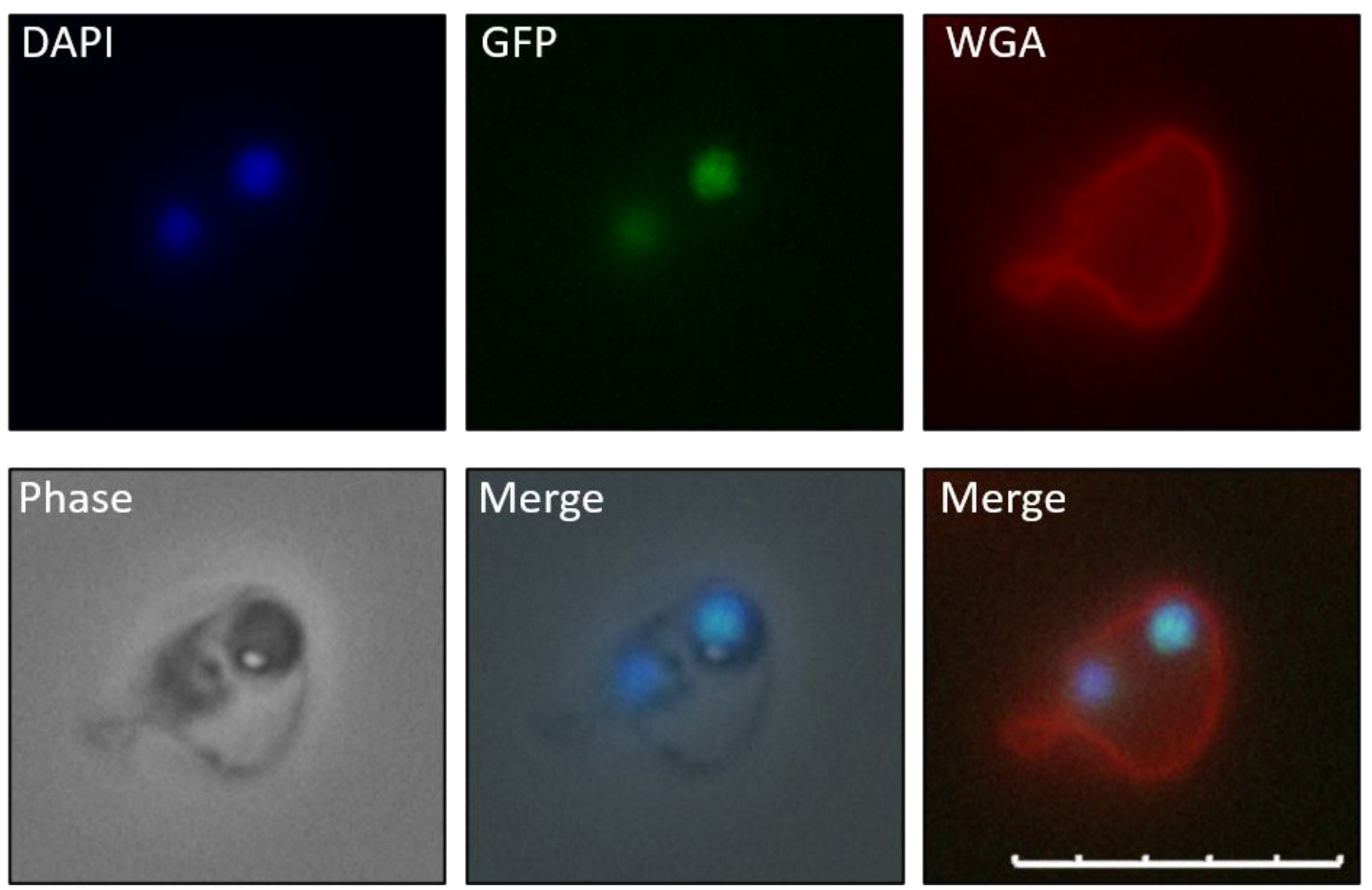

Figure S2 - Fluorescence microscopy of ring stage $P$. falciparum parasites exhibiting GFP expression. DAPI was used as parasite nuclear marker and WGA as erythrocyte surface marker. Scale bar: $10 \mu \mathrm{m}$.
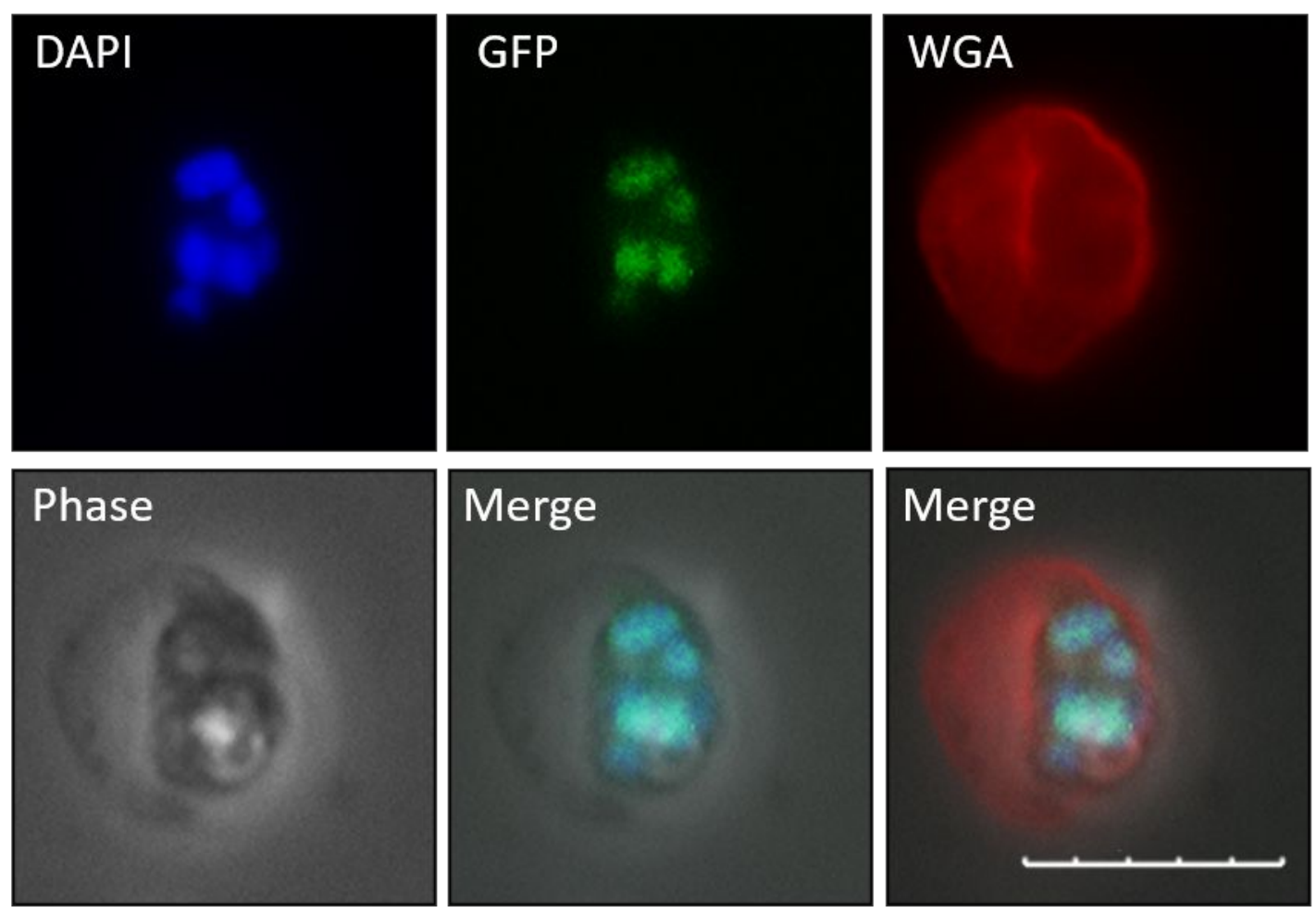

Figure S3 - Fluorescence microscopy of late trophozoite stage $P$. falciparum parasites exhibiting GFP expression. DAPI was used as parasite nuclear marker and WGA as erythrocyte surface marker. Scale bar: $10 \mu \mathrm{m}$. 


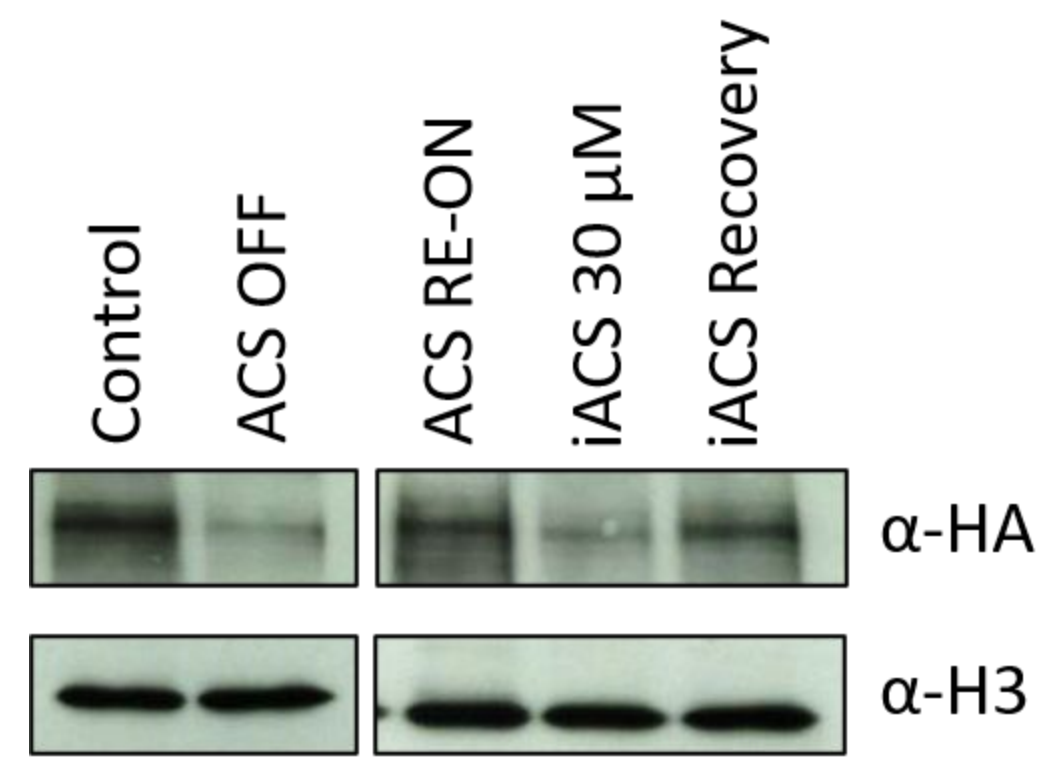

Figure S4 - Western blotting indicating PfACS knockdown (ACS OFF) and, after knockdown treatment (Shield-1 removal plus $10 \mathrm{mM}$ glucosamine/HCl), PfACS is reappearing (ACS RE-ON). On the right side, treatment with $30 \mu \mathrm{M}$ of the iACS inhibitor leads to decreased amounts of HA tagged PfACS, which returns to normal levels when the inhibitor is washed out (iACS recovery). 


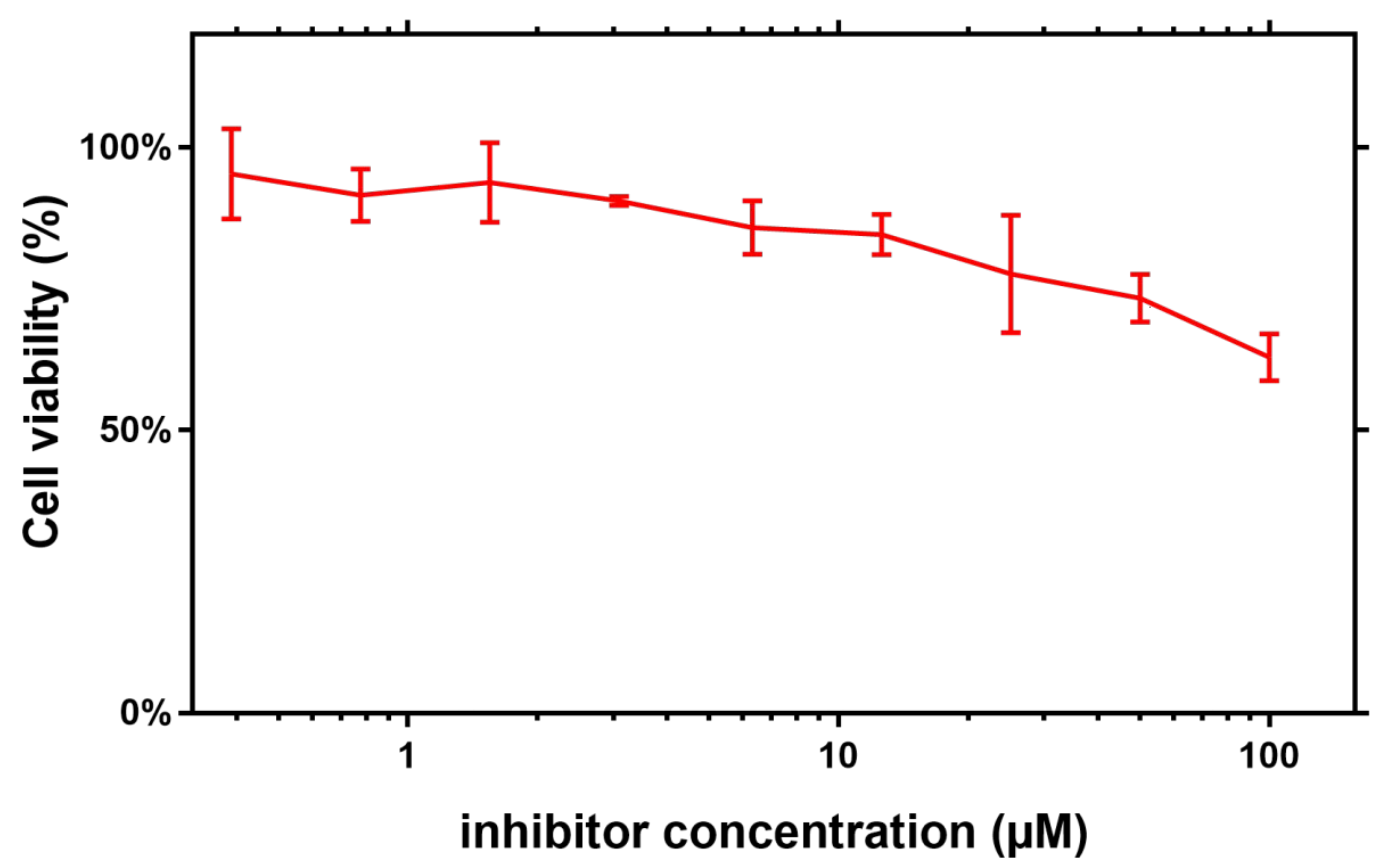

Figure S 5: Cytotoxicity of Ac-CoA Synthetase iACS tested in Hep G2 cells. Cell viability was assessed by measuring mitochondrial activity using WST-1. Assay was conducted once with technical triplicates in a range from 100 to $0.39 \mu \mathrm{M}$. Values are shown as means including SD (error bars). For this test, Hep G2 cells (ATCC® HB8065 ${ }^{\mathrm{TM}}$ ) were maintained in Dulbecco's modified Eagle medium (DMEM, Atena Biotecnologia; Campinas, Brazil) supplemented with 10\% (v/v) FBS, $2 \mathrm{mM}$ L-glutamine, $1 \mathrm{mM}$ sodium pyruvate, and Penicillin/Streptomycin. Cells were cultivated under $5 \% \mathrm{CO}_{2}$ atmosphere at $37^{\circ} \mathrm{C}$ and passaged every $48-72$ hours using $1 \mathrm{x}$ PBS and $0.25 \%(\mathrm{w} / \mathrm{v})$ Trypsin- $0.53 \mathrm{mM}$ EDTA solution. The cytotoxic effect of iACS (MedChemExpress LLC, USA; Cat. No.: HY-104032) was assessed using the cell proliferation reagent WST1 (Roche). Two-fold serial dilutions were prepared in a flat bottom 96-well plate (Sarstedt) in a range from $100 \mu \mathrm{M}$ to $0.39 \mu \mathrm{M}$ and Hep G2 cells seeded at $10^{4}$ cells/well $(100 \mu \mathrm{L})$. Non-treated cells (maximal proliferation), cells treated with the maximal solvent concentration (DMSO; solvent control), medium with the maximal compound concentration (native absorbance), and medium only (background) served as controls. DMSO concentration never exceeded $0.5 \%(\mathrm{v} / \mathrm{v})$. Plates were incubated for 48 hours at $37^{\circ} \mathrm{C}$ and $5 \% \mathrm{CO}_{2}$. After incubation, metabolic activity of cells was measured by adding $10 \mu \mathrm{L}$ WST-1 cell to each well following an additional 2 hours of incubation. WST-1 is a tetrazolium salt and is metabolized by the mitochondrial succinate-tetrazoliumreductase system of living cells and forms formazan which absorbance was measured at $440 \mathrm{~nm}$ using the POLARstar plate reader (BMGLabtech, Germany). Absorbance at 630 $\mathrm{nm}$ was measured as reference wavelength. Data was acquired via the OMEGAstar and MARS software, manually normalized, and plotted in GraphPad Prism (version 7.00 for Windows, GraphPad Software, La Jolla California USA, www.graphpad.com). 